\title{
Transcutaneous Near Infra-Red Spectroscopy (NIRS) for monitoring paediatric renal allograft perfusion
}

\author{
Filippo Ghidini ${ }^{1}$, Elisa Benetti ${ }^{1}$, Pietro Zucchetta ${ }^{1}$, Piergiorgio Gamba ${ }^{1}$, and Marco \\ Castagnetti $^{2}$ \\ ${ }^{1}$ University of Padua \\ ${ }^{2}$ Univeristy of Padua
}

December 14, 2020

\begin{abstract}
Background. The use of transcutaneous near-infrared spectroscopy (NIRS) for the monitoring of the perfusion of renal allografts in paediatric population has been proposed in the last years. This device might detect early decrease in allograft oxygenation allowing prompt detection of vascular complications. Methods. A systematic review of literature about the use of transcutaneous NIRS in monitoring allograft perfusion was performed according to the PRISMA guidelines. Results. The authors screened 1313 papers. The search yielded five pertinent articles. Three of them reported the experience of NIRS in kidney transplantation, for a total of 53 paediatric patients and 50 adults. In these studies, NIRS measurements was significantly related to serum creatinine, estimated glomerular filtration rate (eGFR), urinary neutrophil gelatinase-associated lipocalin (u-NGAL), serum lactate, resistive index assessed by doppler-ultrasonography and systolic blood pressure. In the paediatric studies no vascular complications were encountered. Conclusions. Preliminary studies have related NIRS monitoring to renal allograft perfusion and function. Further investigation is needed to establish the normal range of NIRS values for renal allografts and the factors influencing NIRS monitoring.
\end{abstract}

Short Report

Transcutaneous Near Infra-Red Spectroscopy (NIRS) for monitoring paediatric renal allograft perfusion

Filippo Ghidini ${ }^{1,2}$, Elisa Benetti ${ }^{3}$, Pietro Zucchetta ${ }^{4}$, Piergiorgio Gamba ${ }^{2}$, Marco Castagnetti ${ }^{1,5}$

${ }^{1}$ Paediatric Urology, Department of Women's and Children's Health; University of Padua, Italy

2 Paediatric Surgery, Department of Women's and Children's Health; University of Padua, Italy

${ }^{3}$ Paediatric Nephrology, Department of Women's and Children's Health; University of Padua, Italy

${ }^{4}$ Nuclear Medicine. Department of Medicine; University of Padua, Italy

${ }^{5}$ Department of Surgery, Oncology, and Gastroenterology; University of Padova, Padua, Italy

Corresponding Author : Filippo Ghidini

Department of Women's and Children's Health, University of Padua, Italy

via Nicolò Giustiniani, 35121 Padua, Italy

$+393495313815$

filippo.ghidini@studenti.unipd.it 
Abstract word counts: 177

Main text word counts: 1055

References: 15

Table: 1

Supplementary materials: 2

Running Head Title. NIRS for paediatric kidney transplantation

\section{Abstract}

Background. The use of transcutaneous near-infrared spectroscopy (NIRS) for the monitoring of the perfusion of renal allografts in paediatric population has been proposed in the last years. This device might detect early decrease in allograft oxygenation allowing prompt detection of vascular complications.

Methods. A systematic review of literature about the use of transcutaneous NIRS in monitoring allograft perfusion was performed according to the PRISMA guidelines.

Results. The authors screened 1313 papers. The search yielded five pertinent articles. Three of them reported the experience of NIRS in kidney transplantation, for a total of 53 paediatric patients and 50 adults. In these studies, NIRS measurements was significantly related to serum creatinine, estimated glomerular filtration rate (eGFR), urinary neutrophil gelatinase-associated lipocalin (u-NGAL), serum lactate, resistive index assessed by doppler-ultrasonography and systolic blood pressure. In the paediatric studies no vascular complications were encountered.

Conclusions. Preliminary studies have related NIRS monitoring to renal allograft perfusion and function. Further investigation is needed to establish the normal range of NIRS values for renal allografts and the factors influencing NIRS monitoring.

\section{Keywords}

Near Infra-Red Spectroscopy, kidney transplantation, thrombosis, doppler-ultrasonography

\section{Declarations}

Funding . None declared.

Conflict of Interest . None declared.

Authors' contribution. All Authors contributed to the study conception and design. FG and MC performed the literature search and the screening of the papers. FG and MC wrote the first draft of the manuscript. EB, PZ and PG critically reviewed and commented the previous version of the manuscript. All authors read and approved the final manuscript.

\section{Introduction}

Kidney transplantation (KT) has become the treatment of choice for paediatric end-stage renal disease [1]. Nevertheless, in comparison to adults, the small caliber of the vessels and the common size-mismatch between donors and recipients can predispose to vascular complications including allograft thrombosis. The latter may affect up to $10 \%$ of KTs and account for 35\% of allograft losses in the first year [2]. Prompt recognition and treatment is clearly essential for the preservation of the allograft.

Vascular complications have no specific clinical or biochemical signs allowing for early diagnosis. Dopplerultrasonography (DUS) and renal scintigraphy are reliable tools to assess allograft perfusion [3], but do not allow for continuous monitoring of the allograft and can miss early diagnosis, even if performed with a strict schedule in early follow-up of KT [4]. 
Transcutaneous near-infrared spectroscopy (NIRS) allows for non-invasive, real-time, continuous monitoring of regional oxygenation of the haemoglobin ( $\mathrm{rSrO} 2$ ), which is an indirect measure of the blood flow and the metabolic state, of tissue placed deeper beyond the skin. Several clinical studies have tested the use of NIRS for monitoring cerebral and somatic perfusion in intensive care units [5] and the viability of soft-tissue flaps [6]. Recently, it has been proposed for the surveillance of allograft perfusion too [7].

A systematic search of literature was performed, aiming to identify the current evidence on the in vivo application of NIRS for the monitoring of allograft perfusion. The findings might be useful for future research to implement the large-scale use of NIRS.

\section{Materials and Methods}

The systematic review was performed according to the PRISMA guidelines [8]. PubMed, Scopus and The Cochrane Library were searched in November 2020, using a combination of terms including "near-infrared spectroscopy", "NIRS" and "transplantation". The search was limited to English language and publications from 1991 to 2020. Studies including animal models were excluded.

\section{Results}

Overall, 1313 papers were screened. The search yielded only five pertinent articles (Appenice $A$ ). Three of them investigated renal graft perfusion, accounting for a total of 53 paediatric patients and 50 adult patients. Main findings of the selected papers were reported in the Table below. The quality assessment, according to the Newcastle-Ottawa Scale, showed an high risk of bias for all of them (Appendice B ). This might be due to the small size of the cohorts selected for the studies.

\section{Discussion}

A preliminary study by Vidal et al. reported transcutaneous NIRS measurements in 24 children after KT [7]. Renal rSrO2 measured by NIRS significantly increased over time during the first three days after surgery and a significant correlation was found with serum creatinine, estimated glomerular filtration rate (eGFR) and with the decrease of post-operative urine-neutrophilgelatinase-associated lipocaine (u-NGAL), which is a marker of tubular injury reflecting the ischemic and reperfusion damage of the kidney. In this series, DUS did not identify any abnormality in renal vascularisation and no vascular complications were reported. To date, four patients experienced a delayed graft function (DGF) without any peculiar modifications of rSO2.

Despite these promising results, Skowno et al. raised some criticisms [8]. First, DUS, the gold standard for the assessment of allograft perfusion, was not related to $\mathrm{rSrO} 2$. Recently, Malakasiotiet al . identified a significant correlation between renal rSO2 measured by NIRS and resistive index derived from DUS in a series of 29 paediatric patients [9]. Once again, no complications were reported in the cohort.

Second, the region of interest of NIRS may reach up to $4 \mathrm{~cm}$ below the skin, according to the manufacturers, and its effectiveness in monitoring somatic perfusion was validated in infants weighing less than $10 \mathrm{~kg}$ [10]. This body weight was sensibly lower than those of the patients undergone KT. However, Skowno et al. confirmed the effect of body size on transcutaneous NIRS only in a porcine model, suggesting its in vivo application up to $1 \mathrm{~cm}$ of depth [11].

Nevertheless, Pérez Civantos et al. assessed renal $\mathrm{rSrO} 2$ in 61 adults undergone KT, using probes with a maximum depth of $2.5 \mathrm{~cm}$ [12]. NIRS readings were significantly correlated to the decreasing values of serum lactate at eight hours and 24 hours, which is another marker of ischemic injury. Furthermore, renal $\mathrm{rSrO} 2$ was related to initial diuresis at three hours and to mixed central venous oxygen saturation. On the other hand, no correlation with DUS was found. In addition, in this series arterial thrombosis and bleedings were encountered, showing a maintained decrease in $\mathrm{rSrO} 2$ which might anticipate the vascular events. Moreover, the same study was replicated in liver transplantations. Even in this scenario, NIRS decreased early during bleeding or in case of thrombosis [13].

The last concern about NIRS regarded the placement of the probes. The vascularisation over the surgical wounds may be altered by the healing process, Skowno et al. speculated that a placement directly over that 
area might influence NIRS readings [8]. Therefore, experimental studies on reliable animal models, such as rats or piglets, should better clarify the real impact of abdominal wall thickness and of the healing process on transcutaneous NIRS monitoring.

Furthermore, the normal range of renal $\mathrm{rSrO} 2$ values need to be established by investigating a larger number of patients. Only after this process, NIRS might help in the prompt identification of acute adverse events, which is the final aim of real-time NIRS monitoring. At the moment, NIRS contributed to the early diagnosis of vascular complications in two children undergone liver transplantation [15]. However, even if renal $\mathrm{rSrO} 2$ was not altered among the patients affected by DGF in the series by Vidal et al. , the Authors suggested that NIRS might help in discriminating the causes of oligo-anuria in the early post-operative time after KT, helping in managing the postoperative fluid balance [7].

In conclusion, preliminary studies showed the correlation between NIRS, graft function and graft perfusion in paediatric KTs. Further investigation should also relate $\mathrm{rSrO} 2$ to perfusion renal scintigraphy, which is the most objective modality to assess renal allograft vascularisation and function [4]. Before large-scale in vivoutilisation, experimentation of NIRS on animal models might investigate factors influencing the measurements of renal $\mathrm{rSrO} 2$, such as the depth of the region of interest and the correct placement of the probes. Furthermore, animal models or selected cohorts of patients might help in defining the normal values of renal $\mathrm{rSrO} 2$.

\section{References}

1. Gulati A, Sarwal MM. Pediatric renal transplantation: an overview and an update. Curr Opin Pediatr 2010; 22(2):189-196.

2. Wang C, Greenbaum LA, Patzer RE, Garro R, Warshaw B, George RP et al. Renal allograft loss due to renal vascular thrombosis in the US pediatric renal transplantation. Pediatr Nephrol 2019; 34(9):1545-1555. Monitoraggio DUS

3. Nixon JN, Biyyam DR, Stanescu L, Phillips GS, Finn LS, Parisi MT. Imaging of pediatric renal transplants and their complications: a pictorial review. Radiographics 2013; 33(5):1227-51.

4. Baxter GM. Ultrasound of renal transplantation. Clin Radiol2001; 56(10):802-18.

5. Denault A, Ali MS, Couture EJ, Beaubien-Souligny, Bouabdallaoui N, Brassard P et al. A Practical Approach to Cerebro-Somatic Near-Infrared Spectroscopy and Whole-Body Ultrasound. J Cardiothorac Vasc Anesth 2019; 33 Suppl 1:S11-S37.

6. Kagaya Y, Miyamoto S. A systematic review of near-infrared spectroscopy in flap monitoring: Current basic and clinical evidence and prospects. J Plast Reconstr Aesthet Surg 2018; 71(2):246-257.

7. Vidal E, Amigoni A, Brugnolaro V, Ghirardo G, Gamba P, Pettenazzo A et al. Near-infrared spectroscopy as continuous real-time monitoring for kidney graft perfusion. Pediatr Nephrol 2014; 29:909-914.

8. Liberati A, Altman DG, Tetzlaff J, Mulrow C, Gøtzsche PC, Ioannidis JPA et al. The PRISMA statement for reporting systematic reviews and meta-analyses of studies that evaluate health care interventions: explanation and elaboration. PLoS Med 2009; 6(7):e1000100.

9. Skowno JJ, Karpelowsky JS. Near-infrared spectroscopy for monitoring renal transplant perfusion. Pediatr Nephrol 2014; 29:2241-2242.

10. Malakasioti G, Marks SD, Watson T, Williams F, Taylor-Allkins M, Mamode N et al. Continuous monitoring of kidney transplant perfusion with near-infrared spectroscopy. Nephrol Dial Transplant 2018; 33:1863-1869.

11. Ortmann LA, Fontenot EE, Seib PM, Eble BK, Brown R, Bhutta AT. Use of near-infrared spectroscopy for estimation of renal oxygenation in children with heart disease. Pediatr Cardiol 2011; 32:748-753. 
12. Skowno JJ, Karpelowsky JS, Watts NR, Little DG. Can transcutaneous near infrared spectroscopy detect severe hepatic ischemia: a juvenile porcine model. Paediatr Anaesth 2016; 26(12):1188-1196.

13. Pérez Civantos DV, Muñoz Cantero A, Robles Marcos M, Fariñas Seijas H, Santiago Triviño MA, Pérez Frutos MD et al. Utility of Basal Regional Oximetry Saturation for the Diagnosis of Acute Tubular Necrosis in the Early Postoperative Period Following Kidney Transplantation. Transplant Proc 2019; 51(2):328-333.

14. Pérez Civantos DV, Muñoz Cantero A, Robles Marcos M, Fuentes Morillas F, Cerezo Arias MO, Fariñas Seijas H. Utility of Basal Regional Oximetry as an Early Predictor of Graft Failure After Liver Transplant. Transplant Proc 2019; 51(2):353-358.

15. Shiba J, Satoh M, Taira K, Niwa Y, Inoue S, Mizuta K et al. Near-infrared spectroscopy might be a useful tool for predicting the risk of vascular complications after paediatric liver transplants: Two case reports. Pediatr Transplant 2018; 22(1).

Table. Clinical studies assessing the feasibility of real-time NIRS monitoring of allograft perfusion.

\begin{tabular}{|c|c|c|c|c|}
\hline Study & Country & Design & Population & Main Findings \\
\hline $\begin{array}{l}\text { Vidal et al., } 2014 \\
\text { [7] }\end{array}$ & Italy & Prospective & $\begin{array}{l}24 \text { paediatric } \\
\text { kidney } \\
\text { transplants }\end{array}$ & $\begin{array}{l}\text { rSrO2 correlated } \\
\text { with serum } \\
\text { creatinine, eGFR } \\
\text { and u-NGAL. }\end{array}$ \\
\hline $\begin{array}{l}\text { Malakasioti et al., } \\
2018 \text { [9] }\end{array}$ & UK & Prospective & $\begin{array}{l}29 \text { paediatric } \\
\text { kidney } \\
\text { transplants }\end{array}$ & $\begin{array}{l}\text { rSrO2 correlated } \\
\text { with resistive } \\
\text { index by DUS } \\
\text { and systolic blood } \\
\text { pressure. }\end{array}$ \\
\hline $\begin{array}{l}\text { Shiba et al., } 2018 \\
{[15]}\end{array}$ & Japan & Case Report & $\begin{array}{l}2 \text { paediatric liver } \\
\text { transplants }\end{array}$ & $\begin{array}{l}\text { Decrease in } \mathrm{rSrO} 2 \\
\text { anticipated } \\
\text { vascular } \\
\text { complications. }\end{array}$ \\
\hline $\begin{array}{l}\text { Pérez Civantos et } \\
\text { al., } 2019 \text { [12] }\end{array}$ & Spain & Prospective & $\begin{array}{l}61 \text { adult kidney } \\
\text { transplants }\end{array}$ & $\begin{array}{l}\text { Decrease in rSrO2 } \\
\text { anticipated vascular } \\
\text { complications. } \\
\text { rSrO2 correlated } \\
\text { with serum lactate } \\
\text { and initial diuresis. }\end{array}$ \\
\hline $\begin{array}{l}\text { Pérez Civantos et } \\
\text { al., } 2019 \text { [13] }\end{array}$ & Spain & Prospective & $\begin{array}{l}50 \text { adult liver } \\
\text { transplants }\end{array}$ & $\begin{array}{l}\text { Decrease in } \mathrm{rSrO} 2 \\
\text { anticipated } \\
\text { vascular } \\
\text { complications. }\end{array}$ \\
\hline
\end{tabular}

\section{Appendices}

Appendice A. Systematic Review according to the PRISMA guidelines.

Appendice B. Quality assessment of the selected articles according to Newcastle-Ottawa Scale.

\begin{tabular}{llllllll}
\hline Study & Study design & Selection & Selection & Selection & Selection & Comparability & C \\
& & $\mathbf{1}$ & $\mathbf{2}$ & $\mathbf{3}$ & $\mathbf{4}$ & $\mathbf{a}$ & \\
Vidal et al., 2014 [7] & Cohort & & & $*$ & $*$ & $*$ & \\
Malakasioti et al., 2018 [9] & Cohort & & & $*$ & $*$ & $*$ & \\
Shiba et al., 2018 [15] & Cohort & & & $*$ & & $*$ & $*$ \\
Pérez Civantos et al., 2019 [12] & Cohort & & & $*$ & $*$ & $*$ & $*$
\end{tabular}




\section{Hosted file}

Table NIRS.pdf available at https://authorea.com/users/382843/articles/498718-transcutaneousnear-infra-red-spectroscopy-nirs-for-monitoring-paediatric-renal-allograft-perfusion 